although it has the advantage of being given orally, seems less effective, and ampicillin similarly. Comparative statistical evidence of the value of the various penicillins and of the antibiotics is not available, and would be difficult to obtain, but I have no personal doubt that at present in the acute infection methicillin and chloramphenical are the two most effective drugs.

A major anxiety is the development of bacterial resistance in the child with repeated or persistent infection. This develops sooner or later in all these children and makes the continued prophylactic use of antibiotics unwise.
An antibiotic free from this drawback wouli be of the greatest value: in about 12 childre with cystic fibrosis treated for up to one year with daily fucidin I have as yet had no case of drug resistance. But this may in ang? case not develop with any drug given prophyt lactically for a year or more.

For the pseudomonas there appears to $b \frac{\bar{s}}{8}$ no answer at present, whether the antibiotis is given by mouth, injection or by aerosol.

\section{REFERENCES}

IACOCCA, V. F., Bibinga, M. S., and Barbero, G. (1963): Amer. J. Dis. Child., 106, 315.

\title{
PENICILLINASE RESISTANT PENICILLINS IN THE TREATMENT OF SURGICAL STAPHYLOCOCCAL INFECTIONS
}

\author{
Alexander M. Rutenburg \\ Beth Israel Hospital, 330 Brookline Avenue, Boston 15, Massachusetts
}

THE development of a unique series of synthetic penicillins resistant to hydrolysis by penicillinase has permitted effective control of staphylococcal disease.

We have used four of these new penicillins (methicillin, nafcillin, oxacillin and cloxacillin) in the treatment of about 500 patients with staphylococcal infections, half of whom had failed to respond to therapy with other antibiotics.

Staphylococci resistant to penicillin $G$ alone or along with other micro-organisms were cultured prior to new penicillin therapy in all patients. Patients with well localized areas of suppuration without systemic reaction or without evidence of spreading infection who could be expected to respond to incision and drainage only, were not included. The conditions treated can be summarized under the following classifications:

1. Primary soft tissue infections.

2. Post-operative wound infections.

3. Intra-abdominal sepsis.

4. Infections complicating peripheral vascular disease.

5. Lower respiratory tract infections.

6. Enterocolitis.

7. Septicemia.
8. Osteomyelitis.

9. Miscellaneous infections.

Results were evaluated on a clinical and bacteriological basis. Criteria for a goocs result were: subjective improvement, defero vescence within 48-72 hours, return of the elevated leukocyte count to normal, steriliza $\underline{0}$ tion of an infected nidus such as wound $\vec{b}$ exudate, urine, sputum or blood and wound healing.

If these responses were not elicited the result was considered poor. Seventy-eight per cent of patients with a variety of staphylococcaf infections responded to therapy with the new antistaphylococcal penicillins.

For the purpose of this discussion I should like to deal in greater detail with the use of the new penicillins in the treatment of staphylococcal enterocolitis, septicemia and certain types of soft tissue infections.

\section{Enterocolitis}

Thirteen patients with staphylococcal enteros colitis had received prior broad spectrum anti-o biotic therapy either in the form of tetracycline or neomycin sulfate and phthalylsulfathiazole (Sulfathalidine) for preoperative bowel pre paration. All were dehydrated and hado 


\section{H. R. $\delta$ POST OPERATIVE ENTEROCOLITIS}

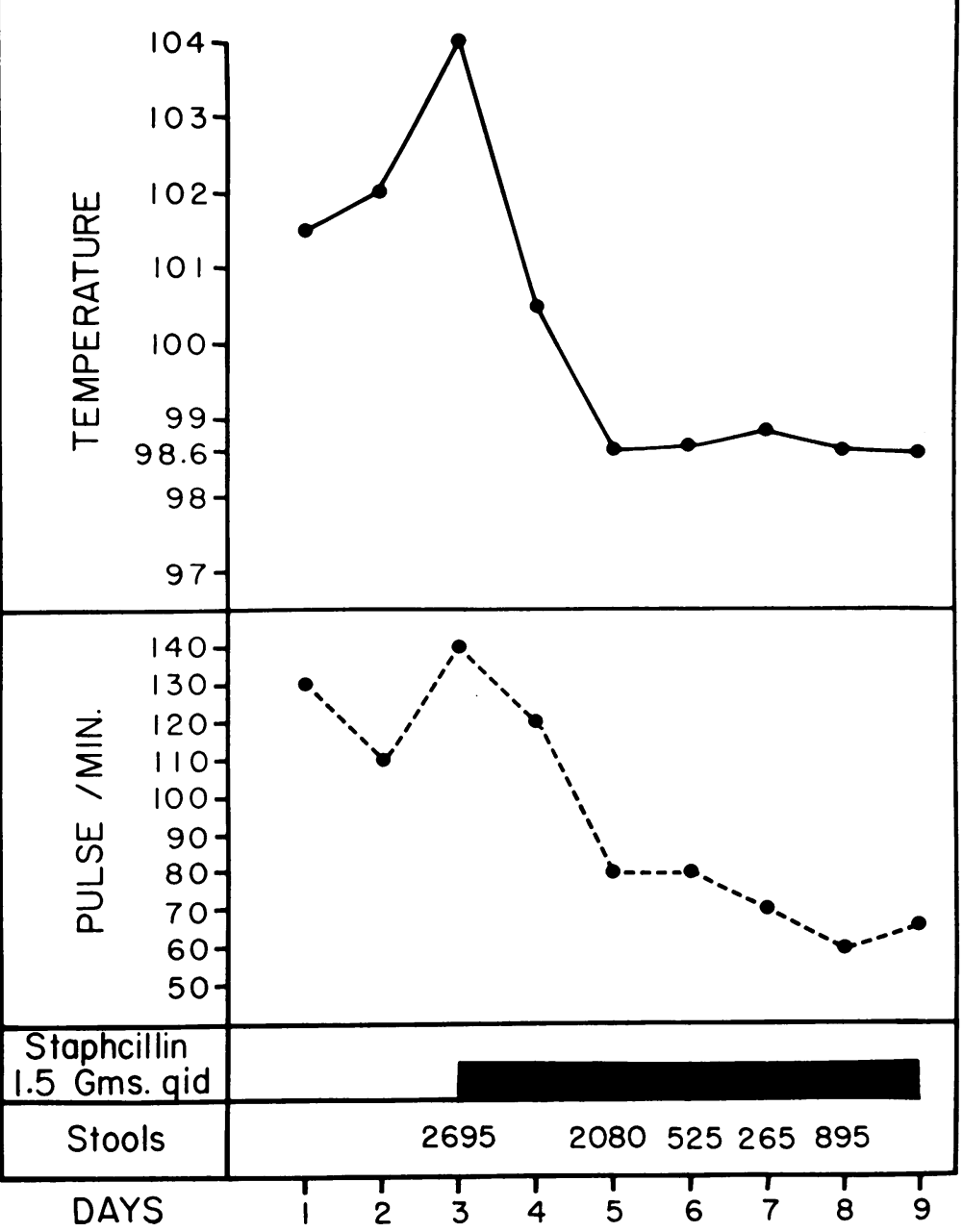

FIG. 1.-Postoperative enterocolitis in a 65-year-old woman.

electrolyte imbalance from profuse, waterydiarrhœa. Clinically they were febrile and had tachycardia, abdominal distension and tenderness. Two patients were hypotensive. Ten patients received oxacillin, two methicillin and one cloxacillin. The duration of therapy ranged from four to 17 days. All 13 patients showed a good response with subsidence of diarrhoea, clinical improvement, eradication of staphylococci from the stools, and return to normal fecal flora.

An illustrative case (Fig. 1) is a 65-year-old woman with profuse, watery diarrhoea, fever of $103^{\circ} \mathrm{F}$., tachycardia, leukocytosis and fluid and electrolyte loss three days after an abdominal operation. Staphylococci in pure culture were identified on stool smear and culture. Tetracycline, which she had been receiving since operation, was discontinued and methicillın (1.5 g every six hours) administered. Within 48 hours she was afebrile, the diarrhoea subsided, and staphylococci were eliminated from the stool. She received methicillin for eight days and was discharged well on the 11 th postoperative day.

Figure 2 shows the result of oxacillin therapy in a patient who developed staphylococcal enterocolitis while receiving neomycin.

Figure 3 shows the postoperative progress of an 84-year-old patient who developed staphylococcal enterocolitis after operation and showed a good response to oxacillin.

\section{Septicemia}

Twenty-one patients with a clinical diagnosis of septicemia received oxacillin. The primary 


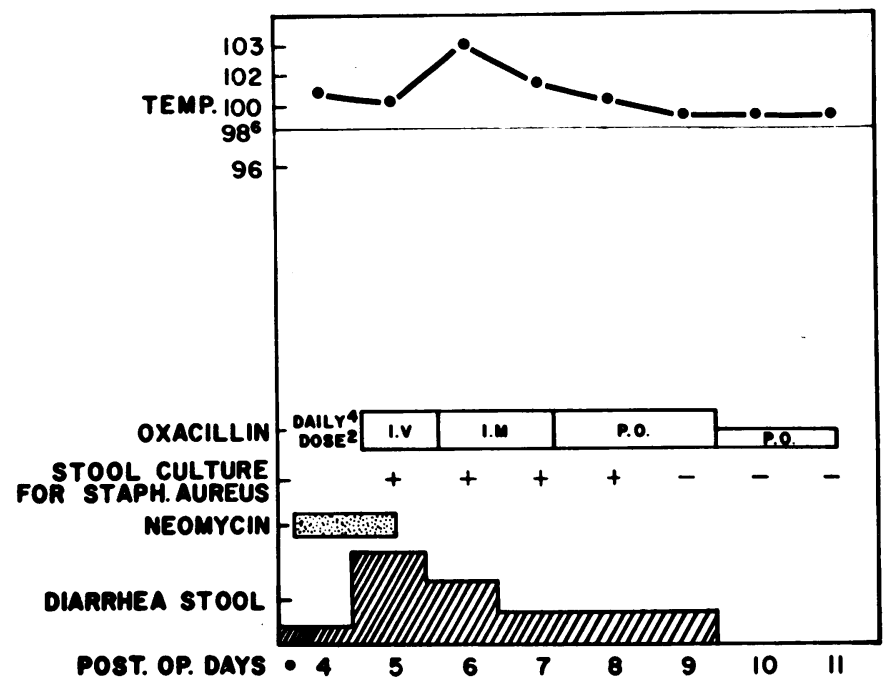

FIG. 2.-Staphylococcal enteritis post porta caval shunt in 53 year old male.

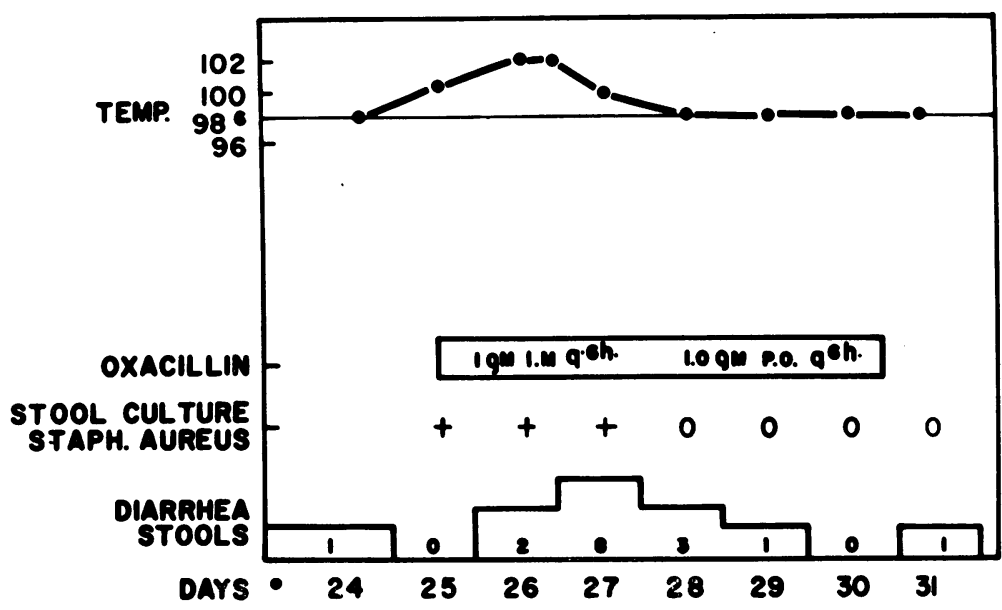

FIG. 3.-Postoperative staphyle coccal enteritis in 84 year ord male.

sites of the infection included the skin, lungs, the wound, an indwelling intravenous polyethylene catheter, deep soft tissue abscess, the heart (endocarditis), and an aortic prosthesis. All were acutely ill with fever, leukocytosis, and other signs of acute systemic infection. Fourteen of the 21 patients had failed to respond to prior antibiotic therapy. In all patients staphylococci were isolated from the blood. The critically ill patients received oxacillin or methicillin intravenously for the first 48 to 96 hours. L arger doses of methicillin than oxacil- lin were required. Subsequently, the drug w\& given intramuscularly $(0.5$ to $1.0 \mathrm{~g}$. every 4 to 6 hours). Four received oxacillin intramusc larly from the beginning of therapy. Duration of therapy ranged from 10 to 20 days in most instances.

Fifteen of the 21 patients responded therapy. The blood was sterilized and the was no recurrence within a six to ten week period of observation. In one patient the result was indeterminate because of concurrent therapy with chloramphenicol. The second 


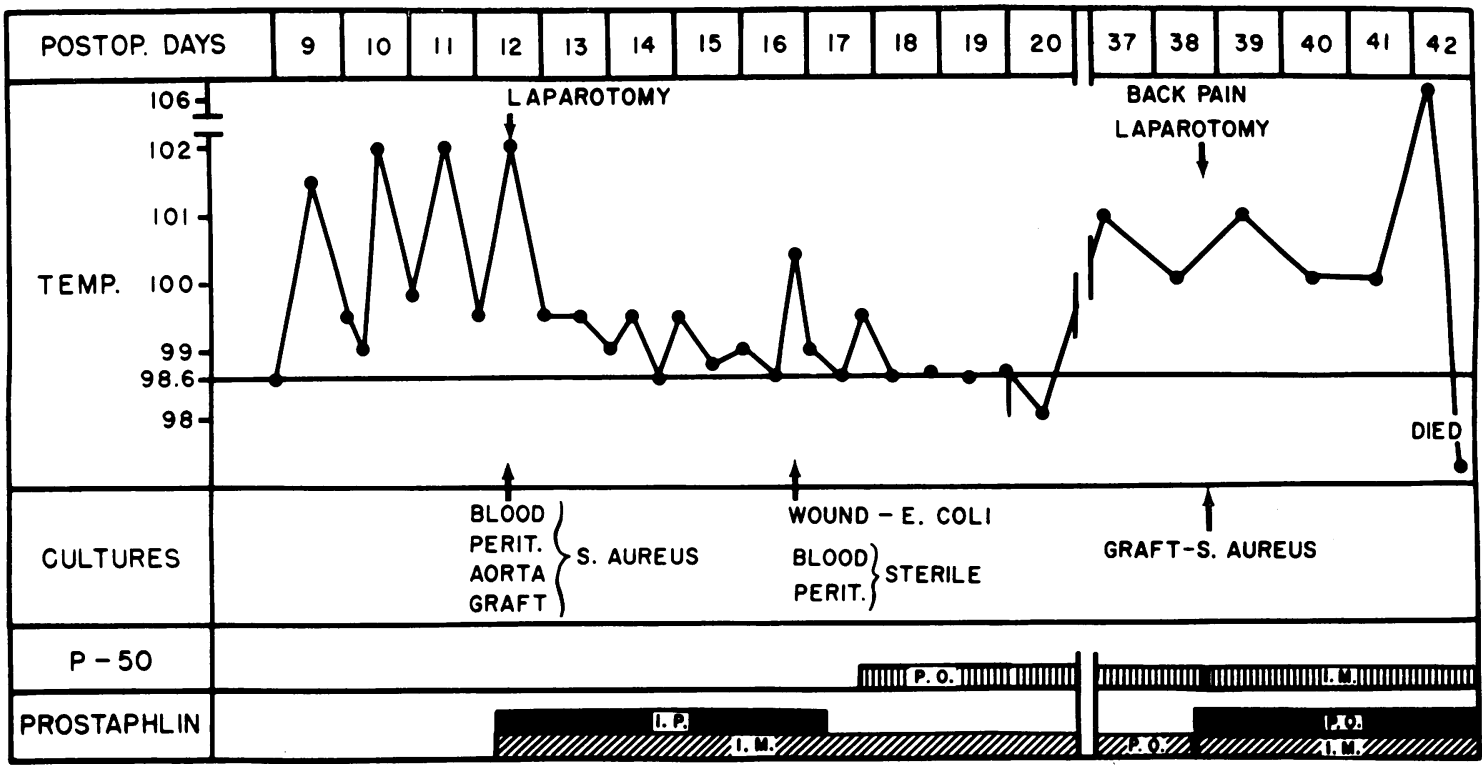

FIG. 4. -60 year old male with septicemia-infected aortic graft.

patient (Fig. 4) developed intermittent fever and leukocytosis nine days after resection of an aortic aneurysm and dacron graft. He was explored because of sudden vascular collapse. At operation he had extensive periaortic and perigraft sepsis with septic intra-aortic thrombosis. Cultures of the peritoneal exudate, aorta, aortic thrombus, and peripheral blood yielded coagulase-positive penicillin G-resistant Staph. aureus which was sensitive to oxacillin (M.I.C. $0.18 \mu \mathrm{g} . / \mathrm{ml}$.). Postoperatively he received oxacillin intramuscularly (3 g.) and intraperitoneally (1.5 g.). His recovery was remarkably prompt and progressive with sterilization of the blood and peritoneal fluid. A wound infection due to Esch. coli was controlled with $\alpha$-amino benzyl penicillin ( 2 to $4 \mathrm{~g}$. daily). He was well until the 37 th day after the exploratory laparotomy when he developed severe back pain. Exploration revealed leakage at the aortic suture line with a huge retroperitoneal hematoma. The graft was replaced with considerable difficulty. Cultures of a segment of the old dacron graft yielded Staph. aureus. This confirmed the familiar contention that infections cannot be controlled nor wounds sterilized in the presence of a foreign body. His subsequent postoperative course was poor. He developed auricular fibrillation, vascular collapse, and died 72 hours later, probably because of myocardial infarction. At post mortem there was no evidence of infection, thrombosis, or leakage at the graft site. A third patient failed to respond because of inadequate therapy. Three patients died within one to four days initiation of oxacillin therapy.

\section{Illustrative Cases}

Case 1. A 64-year-old diabetic with glomerulonephritis developed a staphylococcal urinary tract infection after insertion of an indwelling catheter (Fig. 5). He failed to respond to penicillin $G$, erythromycin, and chloramphenicol given concurrently. He was critically ill with intermittent fever to $104^{\circ} \mathrm{F}$. and coagulase-positive Staph. aureus was cultured from his blood. Methicillin $(20 \mathrm{~g}$./day in divided doses, was given intravenously for two days and intramuscularly (12 g./day in divided doses) for seven days. Thereafter, he showed sterilization of the blood and gradual subsidence of the signs and symptoms of his infection. Methicillin therapy was continued intramuscularly for 25 additional days with a gradual decrease in the daily dose. He was cured after 34 days of treatment with a total dose of $274 \mathrm{~g}$.

Case 2. A 34-year-old male with chronic myelogenous leukemia received corticosteroids, radiation and alkylating agents. He developed extensive softtissue infections with multiple furuncles and carbuncles which involved most of his body surfaces and a septicemia due to Staph. aureus resistant to penicillin G, streptomycin, tetracycline and chlor- 

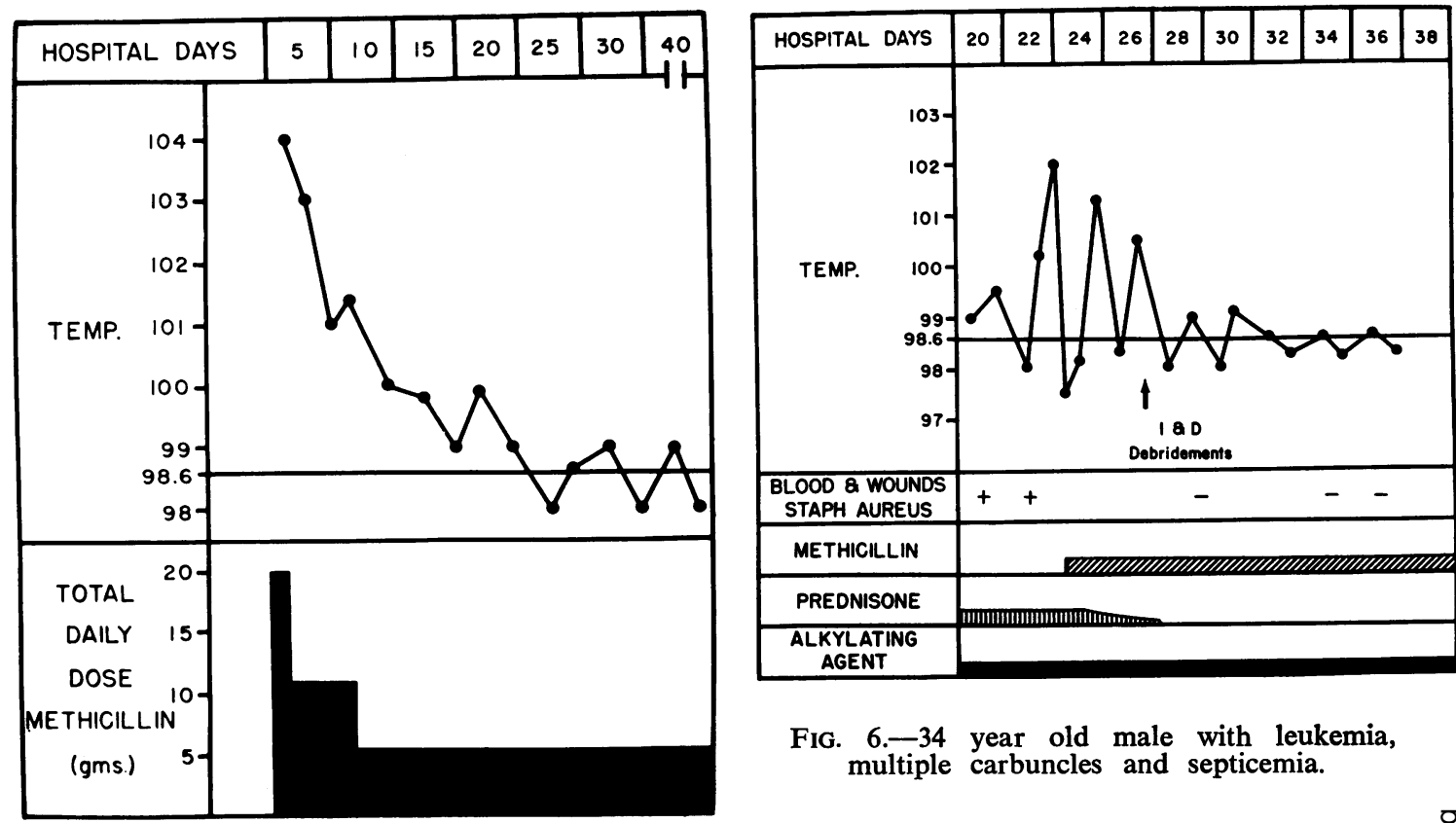

Fig. 6.-34 year old male with leukemia, multiple carbuncles and septicemia.

FIG. 5. -64 year old male with staphylococcal septicemia, glomerulonephritis and diabetes mellitus.

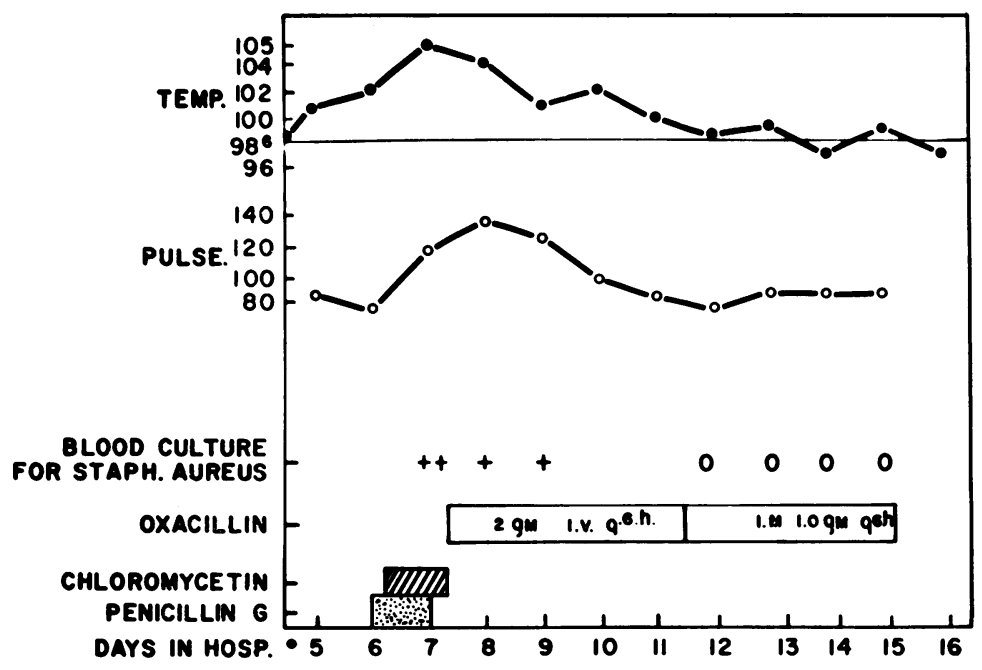

FIG. 7.- Septicemia stemming from an indwelling intravenous catheter in 86 year old female. 


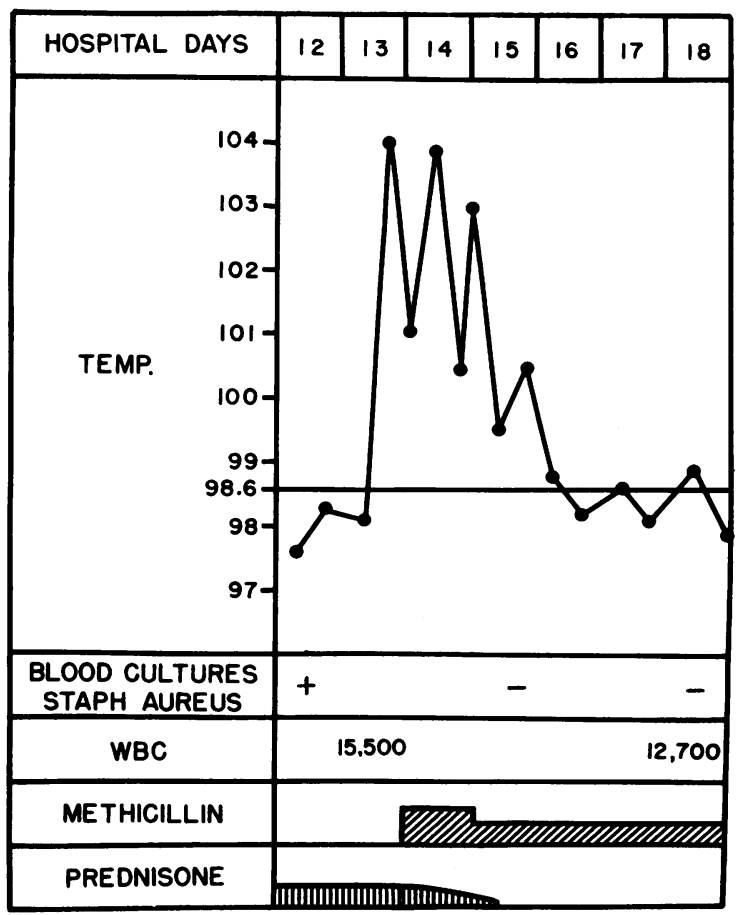

FIG. 8. -61 Staphylococcal septicemia, allergic vasculitis and soft tissue sepsis.

amphenicol. He was in severe pain, febrile and semicomatose. After 24 hours of intravenous methicillin therapy $(8 \mathrm{~g}$ ) his striking improvement (Fig. 6) and control of infection permitted appropriate surgical excision, drainage and debridement of 62 soft-tissue lesions. After six days of therapy, cultures of the wounds, peripheral blood and pharynx were negative for staphylococci. He recovered from his infection after three weeks of methicillin therapy.

Case 3 shows response to oxacillin of a patient with staphylococcal septicemia stemming from indwelling intravenous catheter (Fig. 7).

Case 4. A 61-year-old patient with hypertension and renal failure due to "allergic" vasculitis developed suppurative lesions of the buttock, presacral area and elbow while receiving corticosteroids. Staphylococci were cultured from his wounds as well as his blood. After 24 hours of methicillin therapy (Fig. 8) he showed defervescence and striking control of his infection. Adequate drainage of all soft-tissue lesions was established by excision of suppurative necrotic tissue. Thereafter, he became afebrile and wound cultures were negative for staphylococci. Methicillin was continued for 25 days during which secondary closure of the large soft-tissue defects was carried out successfully.

\section{Comment}

These data indicate the efficacy of methicillin and oxacillin in the treatment of staphylococcal septicemia. The survival of 15 of 21 patients, in many of whom the infection was compounded by underlying chronic disease, is an eloquent testimony of the efficacy of semisynthetic penicillins in this disease. It is possible that the dosage of oxacillin or methicillin administered was greater than the minimum requirement and equally satisfactory therapeutic results could have been achieved with lower dosages. This can be determined by further clinical experience.

\section{Carbunculosis}

A third category of staphylococcal infections treated with methicillin, oxacillin, nafcillin and cloxacillin consists of over 100 patients with furunculosis and carbunculosis. In many, conventional incision and drainage was inadequate and resulted in persistence of local suppuration as well as seeding of satellite lesions and more distant furuncles and carbuncles. A more adequate removal of all necrotic and suppurative tissue accomplished by elliptical excision along with methicillin, oxacillin, cloxacillin or nafcillin therapy resulted in prompt control of the infection. In several instances subsequent skin grafts or closure of the defect with a Z-plasty or full thickness flap were required.

Another illustrative case is that of a 67-yearold diabetic with a history of chronic recurrent furunculosis who developed a giant staphylococcal carbuncle in the left posterior? chest. He had failed to respond to incision and drainage and therapy with penicillin $G$, streptomycin, erythromycin, tetracycline and chloramphenicol. After 24 hours of methicillin therapy the suppurative lesion was widely excised. Methicillin therapy was continued and two weeks thereafter closure of the clean wound was accomplished with the aid of a split thickness skin graft. His recovery was uneventful.

In these patients with recurrent staphylococcal infections new penicillin administration for 15 to 120 days was required. After initial clearance with the first course of therapy, recurrences were seen in about $10 \%$ and these patients required an additional course of therapy. Thereafter, these patients and those who responded to the initial course of therapy remained free from infection for the six to 12 months of follow-up observations.

\section{Discussion}

From the use of these four synthetic penicillins in a large group of patients with various types of staphylococcal infections, the following impressions have emerged: 
(1) All are effective in vitro and in vivo against penicillinase producing staphylococci and most other common gram positive bacteria.

(2) Oxacillin appears to be the most effective agent for intramuscular or intravenous injection in the treatment of infections due to penicillin G-resistant staphylococci. In vitro and pharmacological data suggest that parenteral oxacillin is superior to methicillin largely because of the lower amounts of the drug required.

(3) Cloxacillin is most useful for oral use and yields more regular absorption than oxacillin and nafcillin and more uniform and higher serum levels. It appears to be superior to oxacillin because of better and more uniform absorption after ingestion, lower degradation by the liver and possibly greater resistance to penicillinase hydrolysis than other synthetic penicillins (Bunn and Milicich, 1963; Gravenkemper, Sweedler, Brodie, Sidell and Kirby, 1963).

Although the new penicillins are also effective against certain other gram-positive bacteria such as streptococci and pneumococci on a weight basis, they are less active against these micro-organisms than is penicillin G. Staphylococci resistant to penicillin $G$ required higher concentrations of oxacillin, methicillin, cloxacillin or nafcillin for inhibition than did penicillin $G$ sensitive staphylococci, pneumococci and certain sensitive strains of streptococci. Therefore, the new penicillins are not the drugs of choice for the treatment of infections due to grampositive bacteria other than penicillinaseproducing staphylococci. However, doses recommended for the control of infections due to the latter micro-organisms are sufficiently large to also control infections due to pneumococci, penicillin G-sensitive staphylococci and most strains of streptococci. The new penicillins are ineffective against gram negative bacteria and most strains of Strept. fecalis.

As with other antibiotics the new penicillins are effective in the treatment of surgical infections of the soft tissues if coupled with appropriate surgical management. After removal of foreign bodies, drainage of abscesses, and debridement of necrotic tissue the recovery of many critically ill patients furnished evidence of the considerable efficacy of the supportive value of these drugs. High dosage and prolonged therapy are sometimes required, par- ticularly in septicemia, and certain other recurrent infections, as furunculosis.

The new penicillins demonstrated appareñt efficacy in the treatment of a large series of patients with staphylococcal infections

However, the lack of untreated controls $\mathrm{Fr}$ patients alternately treated with other ant: biotics imposes obvious reservations of defining the relative value of these agents $\stackrel{\mathscr{D}}{\mathbb{D}}$ comparison to certain other antibiotics in the control of staphylococcal infections. On the other hand, of the wide variety of infections treated the satisfactory responses of patients with enterocolitis and septicemia evince the effectiveness of new penicillin therapy. Th many of these patients new penicillin therapy was initiated promptly on the basis of the clinical diagnosis. The infection was also fre quently presumed to be due to staphylococegi on the basis of a smear and a gram stain and subsequently confirmed by culture. Failure to institute prompt therapy can result in thers peutic failure.

Of the patients with severe infections wh did not respond, failure was attributed infections due to resistant strains of grem negative bacteria or Strept. fecalis, death of moribund patient early in therapy, or the presence of an indwelling foreign body undrained sepsis. In a few patients receikng concurrent therapy with other antibiotics, the good response could not be attributed sole to the new penicillin. The prompt response oxacillin, methicillin or cloxacillin therapy all gravely ill patients with staphylococca enterocolitis was particularly striking.

Superinfections with gram-negative bacteria during therapy occurred in patients wit wound infections, occlusive peripheral vascular disease, and infections of the respiratory trace Repopulation of the wounds or sputum with gram-negative bacteria was not always asso 3 ciated with symptomatic superinfection, and treatment with other antibiotics was nos required in all of these patients.

Evaluation of the proper role of the new penicillins in the recovery of patients with sof tissue infections was more difficult because of adjuvant measures such as incision and draino age of localized lesions, debridement, removan of foreign bodies, local heat, etc. Howevern most of these patients were seriously ill with spreading staphylococcal infections and ant to biotic therapy was required to control the extension of the septic process or metastatie spread. 
Staphylococcal strains resistant to oxacillin or cloxacillin were not encountered. Past experience with methicillin, as well as with other antibiotics, has shown that resistant strains occasionally develop following prolonged and injudicious use of these agents. It is possible that the same situation may arise with oxacillin or cloxacillin, although so far no post-therapy-resistant strains have been reported. The useful life of these drugs will be extended by avoidance of routine prophylactic use and inadequate dosage forms and by employing appropriate surgical management of the infection, including adequate drainage, debridement, removal of foreign bodies, etc.

In a few patients gastrointestinal side effects ensued with the larger doses of oral oxacillin and nafcillin but not as yet with cloxacillin. Characteristic "penicillin" type of rash was also observed with all of the new penicillins and therefore, all new semisynthetic penicillins should be administered with care to patients with a history of allergy to penicillin G. On the other hand, of 20 patients previously sensitive to penicillin $\mathrm{G}, 16$ did not react to oxacillin and four did not react to cloxacillin. However, hypersensitivity to penicillin $G$ is sometimes not seen during a repeat course, so that the absence of such symptoms, when oxacillin, given after a previous course of penicillin $\mathrm{G}$ had produced a hypersensitivity reaction, does not always indicate that the patient was hypersensitive to penicillin $G$ and not to oxacillin.

Minimal requirements of therapy are early recognition and bacteriological diagnosis, prompt and adequate antibiotic therapy with an effective agent, and surgical control of the primary foci of infection by drainage, debridement and removal of foreign bodies.

\section{REFERENCES}

BunN, P. A., and Milicich, S. (1963): Antimicrobial Agents and Chemotherapy, Amer. Soc. Microbiol., p. 220.

GravenkeMPer, C. F., Sweedler, D. R., Brodie, J. L., Sidell, S., and KirbY, W. M. M. (1963): Antimicrobial Agents and Chemotherapy, Amer. Soc. Microbiol., p. 231.

\section{DISCUSSION}

\section{H. A. van Geuns (The Hague, Holland).}

I would like to report some results from two clinical trials which my colleague Dr. Kerrebijn and I were running at an out-patient clinic for asthmatic children in Queen Juliana's Children's Hospital in the Hague during the 1962/63 and $1963 / 64$ winters.

Apart from children with simple asthmatic attacks we see many children with recurrent bronchial infections especially during the winter months.

In Holland it is a widely used practice to protect such children during the winter months by continuous administration of sulphonamides. However, we found that about one third of all the pneumococci isolated from such patients proved to be resistant to sulphonamides, and this has been confirmed by other Dutch investigators.

In our first trial 75 children were studied, their ages ranging from four to twelve years. They had all suffered for at least a year from recurrent infections of the lower respiratory tract with an allergic basis. The children concerned are all selected cases as we accepted only those patients referred from the general outpatient clinic which were judged to be serious enough to be treated by our special team. All the children were first examined by the E.N.T. specialist, and were vaccinated with influenza virus vaccine.

The patients were distributed at random over three groups of similar age and comparable living conditions. The prophylactic treatment was given each morning before breakfast. Group one consisted of 24 children taking propicillin (125 mgms.), Group two, 26 children taking sulphadimidine (500 mgms.), and Group three 25 children taking a placebo (one tablet each morning). The experiment was organised on a double-blind basis. Every child was examined at least once a month.

The results of the prophylactic treatment were judged mainly from the number and severity of the respiratory infections developed by the children. Infection was judged on the basis of the findings in the sputum, X-ray examination and physical examination. A secondary criterion was the general impression gained by the parents of the child's condition in the period of treatment as compared to that in the previous year or years. 